\title{
Hypothesis about the Motion of Photon in the Space
}

\author{
Jinjun Cheng \\ Department of Energy, Anhui Energy Administration, Hefei, China \\ Email: cdcdcd999@126.com
}

How to cite this paper: Cheng, J.J. (2019) Hypothesis about the Motion of Photon in the Space. Open Access Library Journal, 6: e5907.

https://doi.org/10.4236/oalib.1105907

Received: November 6, 2019

Accepted: November 19, 2019

Published: November 22, 2019

Copyright $\odot 2019$ by author(s) and Open Access Library Inc.

This work is licensed under the Creative Commons Attribution International License (CC BY 4.0).

http://creativecommons.org/licenses/by/4.0/

\section{(c) (i) Open Access}

\begin{abstract}
This paper attempts to reason about the propositions of photons and its movement in space according to the known axiom of the definition, and by some simple logical method. The light is by the space motion photon component. The space motion photon has the energy and the quality. Photon in space along straight line direction movement speed identically equal in $c$. A photon moves in space with uniform helical motion, which is a combination of uniform circular motion and uniform linear motion in which the speed of the linear motion and the linear speed of the circular motion are always equal to c. Photon's Planck energy formula $E=h v=2 \pi \hbar v, \hbar$ is the approximate Planck constant, which is the angular momentum of photons moving along the circumference plane direction mcr, $r$ is the photon along the circumference plane motion radius; $v$ is photon each second completes the circular motion by link speed $c$ the number of times $c / 2 \pi r$, also is the photon frequency. By replacing the $m c r$ and $c / 2 \pi r$ with the Planck energy formula, it may obtain Einstein mass energy formula $E=m c^{2}$. The greater its mass and energy, the smaller the radius of its circular motion, the higher its frequency, and the shorter its wavelength. The lower its mass and energy, the greater the radius of its circular motion, the lower its frequency, and the longer its wavelength. The uncertainty principle is essentially caused by our misunderstanding of the motion of light. The existence and motion of light can be determined.
\end{abstract}

\section{Subject Areas}

Theoretical Physics

\section{Keywords}

Photons Space Uniform Helical Motion 


\section{Definition}

\subsection{Light}

Visible light is a part of the red to purple range of the electromagnetic radiation spectrum, the definition of light includes all electromagnetic radiation, including infra-red and ultraviolet rays.

\subsection{Photon}

Planck's quantum supposition: between the material oscillator and the radiation energy exchange is not continual, but is one by one, each part can only take an integer multiple of a minimum value. This light of energy is called a quantum of light, also called a photon.

\subsection{Speed of Light}

The speed of light is defined as the photon in the vacuum along the straight line direction dissemination speed, indicated with $c(c=299792456 \mathrm{~m} / \mathrm{s})$.

\subsection{Photon Quality}

The space motion photon has quality, also called the photon movement quality.

\subsection{The Static Quality of the Photon}

The quality of the photon, when the space movement is stopped.

\section{Axiom}

\subsection{The Speed of Light Is Constant Equal to $c$, Independent of the Observer's Motion State}

Michaelson-Morey experiments show that the speed of light is constant equal to $c$ in different inertial systems and in different directions.

\subsection{The Light Has the Granule Nature and the Undulatory Property}

Photoelectric effect, quantum theory and so on to prove that light has particle nature; double slit experiment, Maxwell electromagnetic wave theory proves that the light has the frequency of radiation (hereinafter referred to as frequency), wavelength and other fluctuations.

\subsection{Movement Photons Have Energy}

According to the photon energy formula $E=h v, h$ is the Planck constant, $v$ is the photon frequency, also $v>0$, therefore movement photons have energy. $E>0$.

\subsection{The Frequency and Wavelength of the Photons Are Variable}

Compton experiments show that the frequency and wavelength of photons can change when they collide with material entities. 


\subsection{The Static Quality of Photons Equals 0}

According to restricted theory of relativity, because the speed of light in a vacuum equals $c$, the stationary quality of photons is 0 .

\subsection{Photons Are Self-Rotating}

Self-rotating is the intrinsic property of elementary particles, and photons are the boson of spin 1.

\section{Propositions}

\subsection{Photon Qualities Are Bigger than 0}

By Einstein quality energy conversion formula: $E=m c^{2}$, photon quality $m=E / c^{2}$; by Axiom 2.3, space motion photon energy $E>0 ; c$ is a constant, therefore $m>0$. Therefore, the space motion photon quality is bigger than 0 .

\subsection{Different Frequencies of Photon Energy Are Different}

Supposition photon A and the photon B frequency respectively is $v_{1}$ and $v_{2}$, also $v_{1} \neq v_{2}$; according to Planck energy formula $E=h v$, photon A energy $E_{1}=h v_{1}$ photon B energy $E_{2}=h v_{2}$; because $v_{1} \neq v_{2}, h$ is the constant, therefore $E_{1} \neq E_{2}$. So the different frequency photon energy is different.

\subsection{The Photon Frequency Changes, and the Energy of the Photon Changes with It}

Supposition the photon and the entity collide, before and after the collision frequency respectively is $v_{1}$ and $v_{2}$, also $v_{1} \neq v_{2}$; according to photon energy formula $E=h v$, before photon collision energy $E_{1}=h v_{1}$, after photon collision energy $E_{2}=h v_{2}$. Because $v_{1} \neq v_{2}, h$ is the constant, therefore $E_{1} \neq E_{2}$. So the photon frequency changes, photon energy also along with it change.

\subsection{Different Frequency Photons Have Different Quality}

The supposition photon A frequency is $v_{1}$, the photon B frequency is $v_{2}$, also $v_{1} \neq v_{2}$; by Einstein quality energy conversion formula: $E=m c^{2}$, photon quality $m=E / c^{2}$; Photon A quality $m_{1}=E_{1} / c^{2}$, photon B quality $m_{2}=E_{2} / c^{2}$; according to Proposition 3.2, the photon energy of different frequencies is different, $E_{1} \neq E_{2}$. Because the velocity $c$ of different photons is constant, therefore $m_{1} \neq m_{2}$; So different frequency photons have different qualities [1] [2].

\subsection{Photon Frequency Changes, and the Quality of the Photon Changes with It}

Supposition the photon and the entity collide, before and after the collision frequency respectively is $v_{1}$ and $v_{2}$, also $v_{1} \neq v_{2}$; according to Proposition 2.3, the photon and the entity collide, if the frequency changes, then the photon energy $E$ also changed, $E_{1} \neq E_{2}$. By Einstein quality energy conversion formula: 
$E=m c^{2}$, photon quality $m=E / c^{2}$; because $E_{1} \neq E_{2}$, speed of light $c$ is a constant, therefore, $m_{1} \neq m_{2}$. Therefore, the photon frequency changes, the photon quality also changes.

\subsection{The Stationary Energy of Photons Is 0}

By Axiom 2.5, the stationary quality of photons is 0 , according to Einstein quality energy conversion formula: $E=m c^{2}$, speed of light $c$ is a constant, so the stationary energy of the photon (that is, the energy of the photon stopping the space motion) is also 0 .

\section{Hypothesis}

Photon in space along straight line direction movement speed identically equals in $c$. The photon space motion trajectory is a uniform helical linear motion, which is a combination of uniform circular motion and uniform linear motion in which the speed of the linear motion and the linear speed of the circular motion are always equal to $c$.

The proof is as follows:

\subsection{In the Photon Space Motion Way Contains the Circular Motion}

Photon energy $E=h v$, because the dimensional unit of the Planck constant $h$ is "J.S", and is also the unit of angular momentum, therefore, the energy $E$ of the photon contains the product of the angular momentum of the photon space motion and its motion frequency. Angular momentum is the product of the displacement of an object to its original point and its momentum, is a measure of the circular motion of a particle around its origin. Because in the space motion photon energy contains throughout has the angular momentum, therefore in the photon space motion way contains the circular motion inevitably throughout.

\subsection{Photon Space Motion Trajectory Is the Combined Motion of Circular Motion and Linear Motion}

1) The known space motion photon contains the linear motion mode; it is proved by 4.1 that the photon contains a circular motion in the same way. By Axiom 2.6, the photon has a spin motion, so the final trajectory of the photon space motion should be the combined motion of the above three different motion modes.

2) Photons have spin, but there is no friction in the vacuum. Therefore, the spin motion of photons cannot influence the trajectory of photon space motion.

3) The known photon in the space along the straight line direction movement speed permanent is $c$, meanwhile by proved 4.1 may know, the photon has contained the circular motion way throughout in the space motion. Therefore, the photon space motion path should be a result which the circular motion and the uniform linear motion mode together. 


\subsection{The Direction of the Photon Linear Motion Is Perpendicular to Its Circular Motion Plane}

1) By proved 4.2 may know: the photon space motion path should be a result which the circular motion and the uniform linear motion mode together, and the linear direction of movement speed is equal to $c$. Therefore, the photon space motion can be imagined as: the photon makes the circular motion by certain link speed, forms a circumference plane; simultaneously the circumference plane makes the translational motion by constant speed $c$ along the straight line direction. It is necessary to prove that the circular motion plane is perpendicular to its linear direction of motion.

2) Because the photon makes the circular motion, therefore, the photon receives centripetal force $F$ inevitably throughout in the circular motion plane the function; and because the direction of the photon linear motion is uniform motion, therefore, there is no force in the direction of the linear motion.

3) Suppose that the circular motion plane of the photon does not remain perpendicular to its linear motion, the photon circular motion plane and the linear motion direction are presented in an angle $\theta$, then the photon receives centripetal force $F$ inevitably throughout in the linear motion direction the force component $F \cdot \cos \theta$ function.

If the photon uniform motion is affected by this force, there are two possible situations: one kind of possibility is this force component $F \cdot \cos \theta$ influence photon uniform rectilinear translation; another one possibly does not affect the photon uniform rectilinear translation, but can change the photon unceasingly the frequency (equivalent to photons constantly colliding with solids, Axiom 2.4 Compton experiment).

4) Because the photon maintains the uniform rectilinear translation in the space along the straight line direction, simultaneously the photon energy also maintains invariably, therefore the photon receives circumference centripetal force $F$ the force component $F \cdot \cos \theta$ is inevitably 0 , so $\theta$ is a $90^{\circ}$ angle. So the direction of the photon linear motion is perpendicular to its circular motion plane.

\subsection{The Kinetic Energy of a Photon Is the Sum of Its Kinetic Energy along a Straight Line and the Kinetic Energy of the Motion along a Circular Plane}

By proved 4.2 may know, the spatial motion of photons consists of two sub-motions of linear motion and circular motion; by proved 4.3 may know, the direction of the photon linear motion is perpendicular to its circular motion plane, thus the two sub-motions of the photons moving along the straight line and along the circular plane are independent and not overlapping, so the kinetic energy of a photon is the sum of its kinetic energy along a straight line and the kinetic energy of the motion along a circular plane.

\subsection{The Energy of the Photon in the Space Motion Is All Shown as the Kinetic Energy of the Photon}

The supposition quality is the $m$ material, $v$ makes the uniform rectilinear trans- 
lation by the speed, then the kinetic energy of the linear motion of the substance $0.5 \mathrm{mv}^{2}$, the total energy of the substance shall be the sum of its stationary energy $m c^{2}$ and its kinetic energy $0.5 m v^{2}$. By Proposition 3.6, the stationary energy of the photon is 0 ; by hypothesis 4.4, the kinetic energy of a photon is the sum of its kinetic energy along a straight line and the kinetic energy of the motion along a circular plane. So the energy of the photon in the space motion is all shown as the kinetic energy of the photon.

\subsection{The Movement of the Photon along a Circular Plane Is Uniform Circular Motions}

Because of the conservation of angular momentum of photons moving along the circumference plane, so there are only two possible ways in which photons move along a circular plane: elliptical motions or uniform circular motions.

If it is elliptical motion; Supposes the photon quality is $m, v$ is the photon elliptic motion link speed, $r$ is the photon is apart from the ellipse center the distance, then the photon angular momentum is mvr. In the absence of collisions with other entities, the angular momentum of the photon elliptical motion is conserved. The photon quality, the frequency and the energy also are invariable. Because of the conservation of $m v r$, the velocity $v$ of the photon elliptical motion is bound to change with the $r$ change in inverse proportion, because the kinetic energy of the photon elliptical motion $0.5 \mathrm{mv}^{2}$ changes with time. By proving 4.5 , it is known that the energy $E$ of the photon all shows the kinetic energy of the photon space motion: $E=0.5 m v^{2}+0.5 m c^{2}$. The conclusion is that the energy $E$ of the moving photon is constantly changing without collision with other entities. Since this hypothesis is not true, the motion of photons in the direction of the circumferential plane cannot be elliptical motion, but only uniform circular motions.

\subsection{The Photon along the Circumference Plane Direction Movement Link Speed Permanent Is $c$}

The known photon along the straight line direction movement kinetic energy is $0.5 m v^{2}$, the photon energy is $E=m c^{2}$; Supposes the photon along the circumference plane direction uniform motion link speed is $v$, Then the photon uniform circular motions velocity kinetic energy is $0.5 \mathrm{mv}^{2}$. By proving 4.4 and proving 4.5 , the total energy of the moving photon is the sum of the kinetic energy of the linear motion and the kinetic energy of the circular motion: $0.5 m v^{2}+0.5 m c^{2}=m c^{2}$, so $v=c$. So the photon along the circumference plane direction movement link speed permanent is $c$.

\subsection{The Trajectory of Photon Space Motion Is a Uniform Helical Linear Motion}

The known space motion photon along the straight line direction is the uniform rectilinear translation, the speed is $c$, at the same time the photon in the space also has the uniform circular motions, the line speed is also $c$, and the direction 
of the photon linear motion is perpendicular to its uniform circular motions plane. Therefore, the final trajectory of the photon in space is a uniform helical linear motion, line speed constant $\sqrt{2} c$ (Photons in the space non-linear direction of motion line speed greater than $c$, does not violate Axiom 2.1). The kinetic energy of the photon's constant helix velocity is the energy of the photon. $E=0.5 m(\sqrt{2} c)^{2}=m c^{2}$.

The radius of the photon moving along the circular plane is the amplitude $r$ of the photon; the number of times the photon completes the circular motion in unit time is the frequency $v$ of the photon; the linear distance between the highest points of the adjacent two amplitudes is the wavelength of the photon $\lambda$. Photon energy $E$ is small, explanation photon quality $m$ is small, frequency $v$ is low, oscillation amplitude $r$ is big, wave length $\lambda$ is long; Photon energy $E$ is big, explanation photon quality $m$ is big, frequencyvis high, oscillation amplitude $\mathrm{r}$ is small, wave length $\lambda$ is short.

\subsection{Photon Radiated Angular Momentum Is Dirac Constant}

Supposes the photon quality is $m, r$ is the photon revolves the center of circle movement the radius, the photon uniform circular motion link speed is $c$, and the photon uniform speed straight line direction velocity of movement is $c$. The angular momentum of photons is $m c r$, the photon completes the time which a time uniform circular motion needs is $2 \pi r / c$, each second may complete $c / 2 \pi r$ the inferior circular motion, that is, the frequency of photons $v=c / 2 \pi r$. Because of $E=h v=m c^{2}, v=c / 2 \pi r$, therefore $h \cdot c / 2 \pi r=m c^{2}$, therefore $h=2 \pi \cdot m c r$, photon angular momentum $m c r=h / 2 \pi=\hbar$.

So the photon uniform circular motion conservation of angular momentum, for melts the Planck constant approximately $\hbar$, also is called the Dirac constant. The Planck energy formula can also be changed to: $E=h v=2 \pi \hbar v$.

The trajectory of the photon uniform helical linear motion in space is shown in Figure 1.

The $\mathrm{x}$-axis, $\mathrm{y}$-axis, and $\mathrm{z}$-axis are perpendicular to each other. The photon uniform circular motions plane is parallel to the plane of the $\mathrm{x}$-axis and $\mathrm{y}$-axis, the $\mathrm{z}$-axis is the photon uniform rectilinear translation direction. The trajectory of photon space motion is a uniform helical linear motion.

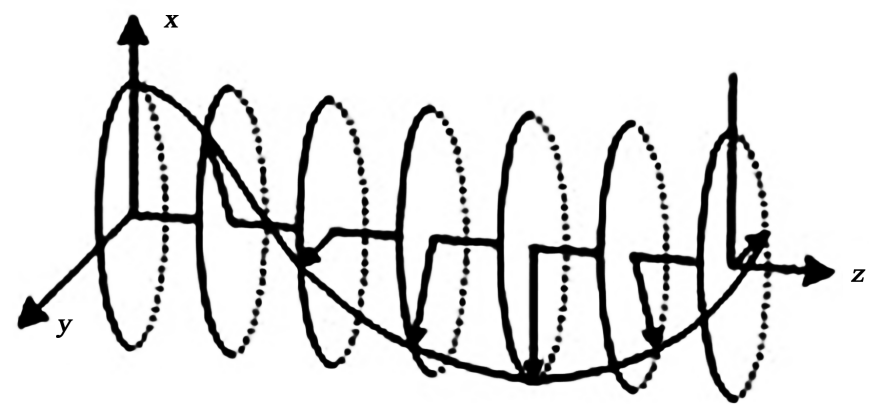

Figure 1. Trajectory of the photon uniform helical linear motion in space. 


\section{Explanations}

\subsection{About the Particle Nature of Light}

The space motion photon has the quality and the energy, can collide with other entities, photons themselves cannot be entered by other entities. Thus, the photons of space motion are also solid particles.

\subsection{About the Fluctuation of Light}

Young's double-slit experiment shows that the light and shade stripes are caused by the collision of the photon with the wall when it passes through the double slit, which makes a part of the photon frequency and wavelength change. The stripe changes the bright place is because projected to this position photon frequency has increased, the wave length shortened, we felt changed shines; The stripe changes the dark place is because projects to this position photon frequency changes small, the wave length changes long, perhaps because the frequency, the wave length become surpass our vision scope to cause.

\subsection{About the Speed of Light Does Not Change}

When a photon collides with the moving entity (the same direction or the opposite collision), the photon energy will increase or decrease. This part of change energy, uses in changing the photon completely the frequency and the wave length, Therefore, the velocity of the photon moving along the straight line is not affected. The photon and the moving entity collide, neither can give the photon linear motion "afterburner", nor give the photon "reduce force", the photon along the straight direction speed constant equals $c$.

\subsection{About the Polarization of the Light}

The photon space motion trajectory is a uniform helical linear motion; its helicoid and the translation direction assume a drift angle, therefore can have the light polarization phenomenon.

\subsection{About the Principle of Uncertainty}

According to Heisenberg, measuring this action inevitably affects the motion state of the particle being measured, thus creating uncertainty. Eure Kennard gives another expression: the uncertainty of position and the uncertainty of momentum are the temperaments of particles; they abide by a certain limit relation, which is independent of the measure action. For the principle of uncertainty, there are two completely different expressions, but the same formula: $\Delta x \Delta p \geq \hbar / 2$ ( $\Delta p$ for momentum survey error, $\Delta x$ for position measuring error, $\hbar$ is Dirac constant).

Since the spatial trajectory of the photon is a constant helical linear motion, we misjudged its trajectory as a straight line, therefore it has the error to its space position survey, this position error $\Delta x$ is throughout photon axial rotation radius $r$. At the same time, because the photon is regarded as uniform mo- 
tion, so we think its momentum is $m c$, but the linear velocity of the photon constant helix is $\sqrt{2} c$, so the actual momentum of the photon is $\sqrt{2} m c$, therefore the photon momentum survey deviation $\Delta p$ is throughout $(\sqrt{2}-1) m c$. So $\Delta x \Delta p=(\sqrt{2}-1) m c r$.

By proved 4.9 may know, $m c r=\hbar$, so $\Delta x \Delta p=(\sqrt{2}-1) \hbar \approx \hbar / 2$.

On the other hand, due to the measurement of the position and momentum of photons, it is necessary to use another photon de-collision measurement with $\lambda$ wavelength, then the error of the measured photon position must be greater than $r$ (possibly $r+\lambda$ ), therefore, the result of the measurement may be as follows: $\Delta x \Delta p \geq \hbar / 2$.

The principle of uncertainty is essentially the result of deviations in the way we understand the movement of light. The existence of light and the way of movement are determined.

\section{Conflicts of Interest}

The author declares no conflicts of interest regarding the publication of this paper.

\section{References}

[1] Greene, B. (1999) The Elegant Universe. W. W. Norton, New York.

[2] Griffiths, G.J. (2004) Introduction to Quantum Mechanics. 2nd Ed., Prentice Hall, Saddle River, NJ. 